\title{
Impaired physiological thermoregulation in postweaning obese female LA/Ntul//-cp (corpulent)
} rats

\begin{abstract}
Groups of lean and obese female lean, and obese LA/Ntul//cp (corpulent) rats were subjected to measures of thermic responses to feeding, noradrenaline administration, and responses to 14 hours of cold exposure to access their capacity for thermoregulation beginning at 6 to 8 weeks of age. Body weights and adipose tissue mass were greater in the obese than the lean phenotype $(\mathrm{p}=<0.05)$. RMR and the dose related thermic responses to norepinephrine (NE) in lean were greater than in obese. Cold exposure at $4^{\circ} \mathrm{C}$ resulted in decreases in rectal but not core temperature in obese rats, and the thermic responses to 45 minutes of $4^{\circ} \mathrm{C}$ cold exposure on $\mathrm{VO} 2$ were typical but were significantly greater in lean than obese animals at all points measured. Circulating thyroid hormone concentrations were similar in lean and obese rats and $\mathrm{T} 3$ but not $\mathrm{T} 4$ increased dramatically in both phenotypes following the cold exposure, consistent with phenotype- and IBAT-linked changes in T4-5' deiodinase and thyroidal activity in this strain. These thermoregulatory changes are consistent with previous studies and may contribute to phenotype mediated aspects of energy metabolism, storage, and adipose tissue deposition and to a phenotype specific selective epigenetic propensity for the expression of obesity during the early postweaning period.
\end{abstract}

Volume II Issue 6 - 202 I

\author{
Orien L Tulp,' George P Einstein, ${ }^{2}$ Aftab A \\ $\mathrm{Awan}^{3}$ \\ 'University of Science Arts and Technology, Montserrat, BWI \\ ${ }^{2}$ University of Health and Humanities, Virgin Islands, Tortola BVI \\ ${ }^{3}$ Einstein Medical Institute, North Palm Beach, FL, USA
}

\begin{abstract}
Correspondence: Orien Tulp, University of Science Arts and Technology, Montserrat, BWI and the University of Health and Humanities, Virgin Islands, Tortola BVI, and the Einstein Medical Institute, North Palm Beach, FL, USA, Email o.tulp@usat.edu
\end{abstract}

Received: December 15, 2021 | Published: December 28, 2021

Keywords: obesity, thermogenesis, T4-5'deiodinase, thyroidal activity, cold exposure

\section{Introduction}

Obesity occurs in the LA/Ntul//-cp rat strain as the result of a autosomal recessive trait, and the physical attributes of the onset of obesity become readily apparent by their rapidly developing physical characteristics in one quarter of the offspring of heterozygous breeding pairs by 5 to 6 weeks of age. ${ }^{1-4}$ Thermoregulation in rodents including parameters of both diet-induced thermogenesis (DIT) and non-shivering thermogenesis (NST) are active components of the thermogenic responses to diet and environment and contribute to maintaining physiologic energy balance and energy expenditure in rodents and likely in other mammalian species as well. ${ }^{5-7}$ The LA/ $\mathrm{N}-c p$ strain was originally developed by Hansen from a Koletsky x Lister/ Albany cross and established in our laboratory from the $12^{\text {th }}$ backcross of the hybrid strain. ${ }^{8-10}$ The LA/N-cp strain was characterized by Michaelis and others several years ago. ${ }^{1,11-14}$ The obesity in older male and female animals has been characterized elsewhere, and includes significant increases in adiposity and final body weights in the obese phenotype from an early age, accompanied by hyperinsulinemia, hyperamylinemia, impaired adrenergic and thyroidal responses, and other endocrinologic parameters typical of the obese state. ${ }^{1,11-14}$ The plasma insulin concentrations become typical of insulin resistance in the obese phenotype but obese animals have reported to remain euglycemic through their lifespan. ${ }^{3,13-14}$ The primary physiologic or biochemical basis for the onset of obesity in this strain remain unclear however, and likely implicates multiple endocrinologic and physiologic factors. ${ }^{15}$

Early studies showed that the obese phenotype exhibited impairments in both the thyroidal and adrenergic components of nonshivering thermogenesis of approximately equal magnitude. ${ }^{16,17}$ These impairments including elements of both diet-induced and environmentally induced contributions to energy during their growth and development. ${ }^{14,16-20}$ Obesity as it occurs in Western society is associated with chronic dysregulation in energy balance and facets of energy metabolism and is rapidly becoming a major public health problem. Complications of obesity have been reported to place an undue burden on not only the economic costs of treatment but also on the economy of delivery of health care resources and including significant losses of workforce productivity among the most severely impacted individuals due to the progressive development of obesity related cardiovascular, renal and other health issues. ${ }^{21-23}$ The congenic LA/Ntul//-cp rodent strain provides an excellent model to explore fundamental biochemical and physiologic parameters associated with the development of obesity due to the specific pathogen free nature of the model, in addition to the congenic status, where the only known difference between the lean and obese littermates is the expression of the obese trait, whereas all other genetic factors are believed to be identical in both phenotypes from conception going forward. ${ }^{1,3}$

\section{Materials and methods}

Groups of lean and obese littermate specific pathogen free (SPF) LA/Ntul//-cp rats were obtained from the DrexelUniversity colony at 5 weeks of age and placed in plexiglass shoebox cages lined with one inch of pine shavings and free access to Purina \#5012 rodent chow and house water. Animals were maintained on a $241800-0600 \mathrm{hrs}$.) at $24^{\circ} \mathrm{C}$ and $40-60 \%$ relative humidity. Animals were randomly selected for each treatment group in littermate pairs (containing one lean and one obese littermate) via a table of random numbers. Animals were fed Purina rodent chow \#5012, containing $3.34 \mathrm{kcal} /$ gram based on data indicated the certificate of analysis on the lot of Purina Chow used in this study. Animals were randomly assigned to individual groups in biological littermate pairs via a Table of random numbers, thereby maintaining equal numbers of animals and constant environmental conditions in animals of each subset thereafter. Measures of body weight were obtained periodically and immediately prior to each physiologic or other experimental procedure conducted in quietly resting animals on an Ohaus animal balance to the nearest gram. Measures of rectal and core body temperatures. Measures of rectal 
and core body temperatures were obtained with a probe to the anus for rectal temperatures, and $12 \mathrm{~cm}$ from the tip of the thermistor to the anus for the upper colonic temperatures. Inspection indicated that this insertion depth corresponded with an area in the upper right quadrant in the mid colon region and directly beneath the mid-lower aspect of the liver. Measures of $\mathrm{VO}_{2}$ plus $\mathrm{NE}$ and cold exposure. Measures of $\mathrm{VO}_{2}$ were obtained with a WE Collins spirometer apparatus (WE Collins, Braintree MA) equipped with a closed-circuit airtight chamber supported with a $\mathrm{CO} 2$ and moisture absorbent, and a constant veristaltic circulating pump (Manostat, NY, NY) to maintain efficient circulation of the closed chamber apparatus during the measurements. The body weight of the animals was recorded to the nearest gram immediately prior to their placement in the sealed $\mathrm{VO}_{2}$ cannister. The chamber was charged with one liter of room air immediately before the animal was placed in the chamber, and the apparatus allowed 10 to 15 minutes to undergo equilibration before actual measurement were taken. Care was taken to ensure that all animals remained in the chamber for the same duration of time for each phase of their observation to minimize experimental error in the measurements. The decrease in the volume of the system during the observation interval then corresponded with the ml. of oxygen consumed by the animal during the process. The $\mathrm{VO}_{2}$ was expressed as the $\mathrm{ml}$ of $\mathrm{O}_{2}$ consumed per minute per $\mathrm{kg}$ of body weight- $/ 75$ as described previously. ${ }^{24,25}$ For measures of norepinephrine (NE) stimulated thermogenesis, the chamber was opened briefly and an injection equivalent to 100 or 200 $\mu \mathrm{g}$ NE per kg of body weight quietly administered subcutaneously in the mid-dorsal region with a tuberculin syringe fitted with a 25 -guage needle so as to minimize injection stress on the animal. All animals were also subjected to two or more 'rehearsals' prior data collection on an attempt to train, familiarize and acclimatize them to the thermogenesis procedures prior to measurements for record.

Measures of thyroid hormone concentrations and T4-5' deiodinase activity. Measures of Triiodothyronine (T3) and thyroxine (T4) were measured by radioimmunoassay, and measures of tissue T3 generation from T4 measured in tissue homogenates, where the tissue T3 generation was reported as picomoles of $\mathrm{T} 3$ generated per mg of tissue homogenate in a phosphate buffer per hour. Enzyme activities were performed at $\mathrm{pH} 7.4$, set at $37^{\circ} \mathrm{C}$. in a shaking incubator. ${ }^{26}$ Tissue and serum protein content was determined by the Folin-Ciocaultau phenol reagent against a standard curve of protein concentrations. ${ }^{27-30}$ Data were analyzed via standard statistical methods including Student's t rest, ANOVA, Duncan's Multiple Range test and others. ${ }^{31}$

\section{Results}

Descriptive parameters of growth and development. The parameters of body weight and tissue weights are presented in Table 1 and show that the weights of the obese rats were nearly double those of their lean littermates by 8 to 9 weeks of age when these measurements were recorded. Body weights at birth were not significantly different and historically could not be used to differentiate the phenotypes, but by 3 to 4 weeks of age those destined to become obese began to assume a broader physical stance in apparent preparation for the greater weight and mass they will eventually be obligated to support. ${ }^{1,10,24}$ Not surprisingly, the liver weights in the present study were already proportionately greater in the obese phenotype but the ratio of liver weight to body weight was similar in both phenotypes, at $2.80 \%$ of body weight in the lean phenotype and $3.01 \%$ of body weight in the obese phenotype but with early signs of imminent fatty liver involvement in the obese phenotype. The Gastrocnemius muscle was dissected as a representative muscle tissue, and indicated that while the absolute muscle weights were similar in both phenotypes, when expressed as a percent of body weight, the muscle weights of obese rats tended to be less than observed in the lean rats (Lean $0.45 \%$ of BW vs Obese $0.37 \%$ of BW, suggesting that lean tissues of grew at a similar rate in both phenotypes, although the data cannot demonstrate differences in the energetic economy of tissue accretion of lean tissues, and is likely to be to be more energetically efficient among obese were the obese animals to have developed early signs of insulin resistance by this age. Mean absolute kidney mass was also greater in the obese than the lean phenotypes likely reflective of developing a greater vascular mass to support their greater body mass, but also reflected a significantly lower percentage of total body weight in the obese phenotype $(0.73 \%$ in lean vs $0.54 \%$ in obese), reflective of other lean, non-adipose tissues.

Table I Weight in grams/depot or tissue in lean and obese rats

\begin{tabular}{|c|c|c|c|c|c|c|c|c|}
\hline Phenotype & $\mathbf{N}$ & Body Weight & Liver & Kidney & GN muscle & RP-AT & Dorsal ISAT & IBAT \\
\hline Lean & 12 & $216.4 \pm 4.7$ & $6.06 \pm 0.33$ & $1.58 \pm 0.04$ & $1.48 \pm 0.04$ & $0.81 \pm 0.10$ & $0.33 \pm 0.04$ & \\
\hline Obese & 12 & $420.5 \pm 11$ & $12.64 \pm 0.59$ & $2.29 \pm 0.11$ & $1.55 \pm 0.06$ & $\mid 5.94 \pm 1.30$ & $162 \pm 0.88$ & \\
\hline$P=$ & ns & $<0.001$ & $<0.001$ & $<0.001$ & $<0.370(\mathrm{~ns})$ & $<0.001$ & $<0.001$ & $<0.001$ \\
\hline
\end{tabular}

Data are mean + I SEM, n, I2 animals/phenotype, 9 weeks of age at time of measurements. $\mathrm{P}$ value determines by $\mathrm{t}$ test. ns= not significant, $\mathrm{p}>0.05$. GN muscle, gastrocnemius muscle. RP, retroperitoneal adipose tissue depot; dorsal ISAT, dorsal interscapular subcutaneous (white) adipose tissue; IBAT, interscapular brown adipose tissue depot. All AT and other tissue depots were dissected in their entirety

Adipose tissue depots. The relative mass of adipose tissue depots differed significantly between the lean and obese phenotypes however, however, and the total mass per depot weights of obese animals was found to be significantly greater than in the lean phenotype in all adipose tissue depots measured. The most prominent differences were noted in the abdominal retroperitoneal depots, where the adipose tissue mass of obese rats was nearly 20 -fold greater in this central depot than occurred in their lean littermates consuming the same diet and subjected to the same environmental conditions throughout. The dorsal depot is reflective of subcutaneous adipose tissue and averaged only 4.9-fold greater mass in the obese than in their lean littermates, suggestive of a differential partition for modulating lipid accretion and energy deposition in central vs. subcutaneous adipose depots in the corpulent phenotype at this age. The Interscapular brown adipose tissue mass, a contributor to energy expenditure in response to alterations in diet and environment in rodents and other mammalian species, ${ }^{5-7,14,19}$ averaged 3.5 -fold greater mass in the obese than the lean phenotype. Obese animals of this and other strains have been reported to consume more total food than their lean counterparts consistent with their greater mass, and generally proportional to their greater body weight and the greater IBAT mass. This may reflect at least in part a dietary or environmentally mediated compensatory indication of their greater caloric intake in the presence of an improved caloric efficiency reported elsewhere. ${ }^{14,24}$ 
Measures of cold-induced body temperature regulation. Animals were maintained in a controlled environment maintained at 24 degrees C. on a reverse light cycle through the study, and subgroups ( $\mathrm{n}=6$ rats / subgroup) were then moved to a $4 \mathrm{C}$ environment in hanging steel cages for 14 hours to determine if they could maintain body temperatures when subjected to the cold temperature as an indication of their thermoregulatory response to the environment. Core and rectal temperatures were recorded after 14 hours via a rectal thermistor apparatus where the recording end of the thermistor was placed in the anal canal, or the upper medial segment of the colon as described above. As shown in Table 2, rectal and core temperatures were maintained in the cold environment in lean animals, while in obese animals' rectal temperatures were decreased in the cold, indicating a significant phenotype effect $(\mathrm{p}=0.012)$ and a phenotypeenvironmental temperature interaction of an undetermined nature. Core temperatures in obese animals also tended to be modestly lower in the obese than the lean phenotype, also indicative of a phenotype specific effect of unclear origin $(\mathrm{p}=0.0038)$.

Table 2 Effects of 14 hours of cold exposure on rectal and core temperatures of lean and obese rats

\begin{tabular}{llllll}
\hline Phenotype & $\mathbf{N}$ & Exposure Temp. & Body Weight & Rectal Temp, ${ }^{\circ} \mathrm{C}$ & Core Temp ${ }^{\circ} \mathrm{C}$ \\
\hline Lean & 6 & $24^{\circ} \mathrm{C}$ & $220.8 \pm 9.0 \mathrm{~b}$ & $37.60 \pm 0.16 \mathrm{a}$ & $38.93 \pm 0.11 \mathrm{a}$ \\
Lean & 6 & $4^{\circ} \mathrm{C}$ & $212.0 \pm 3.3 \mathrm{~b}$ & $36.78 \pm 0.18 \mathrm{a}$ & $38.67 \pm 0.37 \mathrm{a}, \mathrm{b}$ \\
Obese & 6 & $24^{\circ} \mathrm{C}$ & $428.9 \pm 9.1 \mathrm{a}$ & $37.13 \pm 0.17 \mathrm{a}$ & $37.65 \pm 0.73 \mathrm{~b}, \mathrm{C}$ \\
Obese & 6 & $4{ }^{\circ} \mathrm{C}$ & $412.2 \pm 19.8 \mathrm{a}$ & $34.92 \pm 0.55 \mathrm{~b}$ & $37.43 \pm 0.11 \mathrm{C}$ \\
ANOVA & & & & \\
Recorded Temp of Rat & $\mathrm{P}=$ & $0.298 \mathrm{I}$ & 0.0001 & 0.5372 \\
Phenotype & $\mathrm{P}=$ & 0.0001 & 0.0012 & 0.0038 \\
Interaction & $\mathrm{P}=$ & 0.7421 & 0.0354 & 0.9489
\end{tabular}

Data are mean +1 SEM, $n=6$ rats/group. Groups with different letters are significantly different at $p=<0.05$ by Duncan's Multiple Range Test. Rectal temperature determined at $3 \mathrm{~cm}$ rectal insertion and core (colonic) temperature determined at $12 \mathrm{~cm}$ insertion with a YSI thermistor probe. All animals survived the cold exposure without incident

Table 3 Effects of cold exposure on thyroid hormone concentrations of lean and obese rats

\begin{tabular}{lllllll}
\hline Phenotype & $\mathbf{N}$ & Exposure Temp. & T3, ng/dl & T4, $\mu$ g/dl & \multicolumn{2}{l}{$\begin{array}{l}\text { T3/T4, \% } \\
\text { Protein, } \boldsymbol{\mu g} / \mathbf{d l} \text { * }\end{array}$} \\
\hline Lean & $\mu g / \mathrm{dl}$ & $24^{\circ} \mathrm{C}$ & $118.9 \pm 14.5 \mathrm{~b}$ & $2.77 \pm 0.26 \mathrm{~b}$ & $4.32 \pm 0.39 \mathrm{~b}$ & $7.68 \pm 0.42 \mathrm{a}$ \\
Lean & 6 & $4{ }^{\circ} \mathrm{C}$ & $175.9 \pm 15.3 \mathrm{a}$ & $2.54 \pm 0.26 \mathrm{a}$ & $7.25 \pm 0.84 \mathrm{a}$ & $6.99 \pm 0.19 \mathrm{a}$ \\
Obese & 6 & $24^{\circ} \mathrm{C}$ & $121,2 \pm 17.3 \mathrm{~b}$ & $2.31 \pm 0.36 \mathrm{a}$ & $5.36 \pm 0.36 \mathrm{~b}$ & $7.64 \pm 0.12 \mathrm{a}$ \\
Obese & 6 & $4{ }^{\circ} \mathrm{C}$ & $169.9 \pm 12.6 \mathrm{a}$ & $3.15 \pm 0.45 \mathrm{a}$ & $5.82 \pm 0.67 \mathrm{a}, \mathrm{b}$ & $7.97 \pm 0.30 \mathrm{a}$ \\
ANOVA & & & & & & \\
Temp of Rat & & $\mathrm{P}=$ & 0.0022 & 0.3792 & 0.0102 & 0.5341 \\
Phenotype & & $\mathrm{P}=$ & 0.9029 & 0.8173 & 0.7481 & 0.1081 \\
Interaction & & $\mathrm{P}=$ & 0.7826 & 0.1316 & 0.0505 & 0.0801 \\
\hline
\end{tabular}

Data are mean+I SEM, $n=6$ rats/group. Groups with different letters are significantly different at $p=<0.05$ by Duncan's Multiple Range Test.*Serum protein reported as $\mu \mathrm{g} / \mathrm{dl}(x \mid 0 \mathrm{E}+6)$

Table 4 Tissue T4-5' Deiodinase activity in lean and obese cold exposed rats

\begin{tabular}{lllllll}
\hline Phenotype & $\mathbf{N}$ & Temperature & Kidney & IBAT & Liver & GN Muscle \\
\hline Lean & 6 & 24 & $15.7 \pm 1 . \mathrm{I} \mathrm{a}$ & $22.6 \pm 2.2 \mathrm{c}$ & $6.6 \pm 0.4 \mathrm{a}$ & $5.4 \pm 0.9 \mathrm{a}$ \\
Lean & 6 & 4 & $14.0 \pm 0.7 \mathrm{a}, \mathrm{b}$ & $18.8 \pm 1.3 \mathrm{c}$ & $4.8 \pm 0.5 \mathrm{~b}$ & $3.2 \pm 0.7 \mathrm{a}$ \\
Obese & 6 & 24 & $13.1 \pm 1.2 \mathrm{a}, \mathrm{b}$ & $53.7 \pm 7.0 \mathrm{a}$ & $3.25 \pm 0.3 \mathrm{c}$ & $3.8 \pm 0.9 \mathrm{a}$ \\
Obese & 6 & 4 & $11.3 \pm 0.8 \mathrm{~b}$ & $38.3 \pm 3.8 \mathrm{~b}$ & $3.9 \pm 0.4 \mathrm{bc}$ & $4.8 \pm 1.3 \mathrm{a}$ \\
ANOVA & & & & & & \\
Temperature, ${ }^{\circ} \mathrm{C}$ & $\mathrm{P}$ & & 0.0901 & 0.0324 & 0.1552 & 0.5223 \\
$\begin{array}{l}\text { Phenotype } \\
\text { Interaction }\end{array}$ & & $\mathrm{P}=$ & 0.0123 & 0.0001 & 0.0001 & 0.5223 \\
& & $\mathrm{P}=$ & 0.9682 & 0.1816 & 0.0049 & 0.1049
\end{tabular}

Data are mean +/- I SEM, N, 6 rats/group. IBAT, interscapular brown adipose tissue; GN, Gastrocnemius muscle. T4-5' Deiodinase activity is expressed as total deiodinase activity computed as pmol T3 generated from T4 per hour of incubation per mg of homogenate protein at $37^{\circ} \mathrm{C}$. in vitro. Data with different letters indicate significantly different values as computed by ANOVA and Duncan's Multiple Range Test 
Measures of resting, post-fed, norepinephrine (NE) and coldinduced thermogenesis. Measures of $\mathrm{VO} 2$ in the 3-hour post-fed group, referred to as the residual thermic effect of diet-induced thermogenesis (RDIT) reflected only modest increments over the RMR, equivalent to an $18 \%$ increase in lean rats and a $37 \%$ increase over the RMR in the obese rats (Figure 1). DIT effects are well established man and animals, but the duration of the thermic effects of feeding on postprandial $\mathrm{VO}_{2}$ are unclear. The effects of phenotype on resting and a dose response of norepinephrine stimulated thermogenesis are shown in Figure 2 and again show that when fasting resting $\mathrm{VO} 2$ relative to body surface area at thermal neutrality was greater in the lean than the obese phenotype $(p=0.028)$. This phenotype effect persisted following a dose response schedule of NE, where $\mathrm{VO}_{2}$ increased in both phenotypes but the dose related effects of the higher dose were not demonstrated in the obese rats. Marette et all demonstrated that insulin resistance was a significant factor in the activation of NE-stimulated thermogenesis in BAT in a strain of obese-diabetic rats which share the same $-c p$ trait for obesity (Figures $1 \& 2){ }^{32-34}$

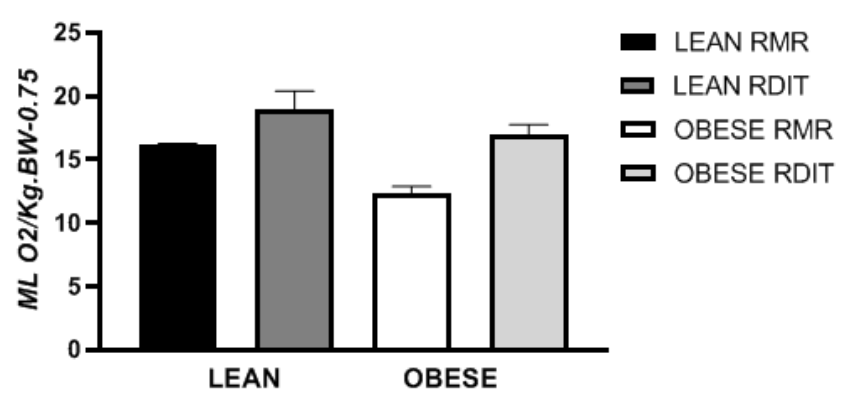

Figure I Post-Fed thermic effect [RDIT] on $\mathrm{Vo}_{2}$

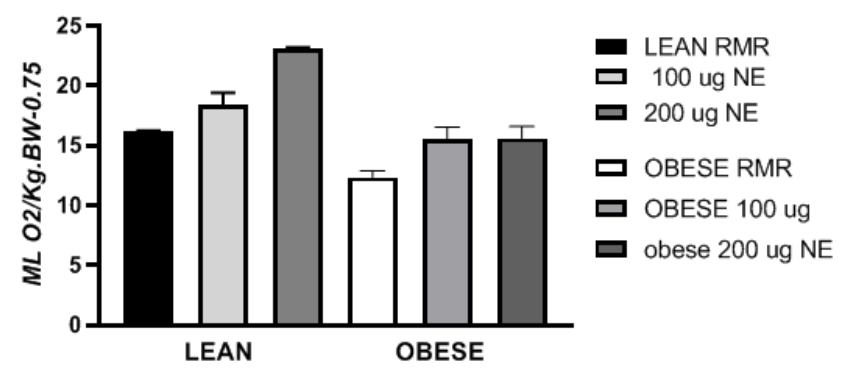

Figure 2 Norepinephrine (NE) administered subcutaneous at 100 or $200 \mu g /$ $\mathrm{kg} \mathrm{BW}$.VO2 is expressed as $\mathrm{ml} \mathrm{O} 2 / \mathrm{kg} \mathrm{BW} / \mathrm{min}^{0.75}$ as described elsewhere. (ref) Rats were removed from food trays for 3 hours prior to post fed measurements as a measure of the residual thermic effect of feeding (RDIT); RMR were fasted for 6 to 8 hours. NE $>$ RMR in lean $(p=<p .05)$; In obese NE at $100 \mu \mathrm{g} / \mathrm{kg}$ BW > obese RMR $(\mathrm{p}=<0.05)$ but the $200 \mu \mathrm{g} / \mathrm{kg}$ BW dose was without additional effect.

Cold-induced thermogenesis. Measures of $\mathrm{VO}_{2}$ in lean and obese rats at thermal neutrality $\left(30^{\circ} \mathrm{C}, \mathrm{RMR}\right)$ compared to 45 minutes at $4^{\circ} \mathrm{C}$ are depicted in Figure 3 and again confirm that resting metabolic rates were greater in lean than obese at thermal neutrality. Measures of $\mathrm{VO}_{2}$ remained greater in the lean than in obese rats at $4^{\circ} \mathrm{C}$ at all times measured up to 45 minutes of continuous cold exposure and stabilized at an intermediate level in lean but not in obese rats by 30 minutes. The initial increase in $\mathrm{VO}_{2}$ at 5 to 15 minutes in both phenotypes is likely a combination of both shivering induced thermogenesis (ST) and nonshivering thermogenesis (NST), while the measures of $\mathrm{VO}_{2}$ observed from 30 minutes going forward are proposed to be more directly linked to the NST than the ST components of adaptive thermogenesis mechanisms of energy expenditure. Because the animals were studied during the late postprandial state, contributions from DIT are likely minor at best. The greater economy of energy expenditure in the obese phenotype is likely to be the result of additive effects of a more efficient capacity for energy conservation as may occur in the presence of greater degrees of insulin resistance common to obesity, decreased rates of oxidative phosphorylation-mediated heat generation in BAT, decreases in the rates of insulinogenically mediated protein turnover, and finally to a greater insulative potential as a result of potential secondary insulative effects of the added adiposity. Surface area relative to mass also may factor into the equation as the ratio of surface area to mass differs significantly between lean and overtly obese animals, and the smaller total mass but a similar lean mass of the lean animals may allow for greater potential for superficial energy dissipation, as the combination of the altered proportion of surface area to body mass ratio in concert with the various metabolic factors suggested above may favor energy expenditure mechanisms toward conservation in obese vs. the lean rats (Figure 3).

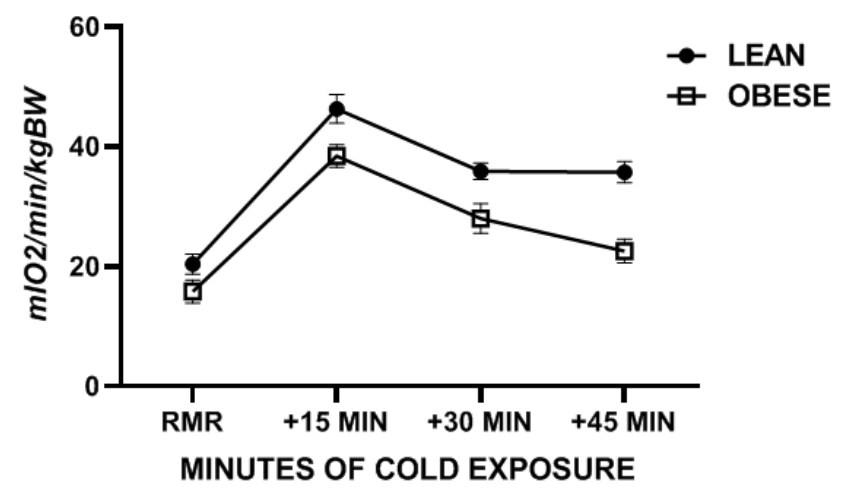

Figure 3 Effect of 45 minutes $4^{\circ} \mathrm{C}$ cold exposure on VO2 in lean and obese $\mathrm{LA} / \mathrm{N} / /$-cp rats. Measures of RMR were obtained at thermal neutrality immediately prior to acute cold exposure. $\mathrm{N}=6-12$ rats/group. ${ }^{*} \mathrm{P}=<0.05$; ** $p=<0.01$.

\section{Discussion}

Obesity occurs in this congenic SPF rodent model as an autosomal recessive trait, likely expressed via an epigenic mechanism as the overall genetics aside from the obese $-c p$ trait is identical in lean and obese littermates, and the multigenerational frequency of obese offspring has consistently remained at $25 \%$ of the offspring of heterozygous breeding pairs for many years. The current strain was derived from the $12^{\text {th }}$ backcross of the Koletsky rat as the source of the $-c p$ trait $^{9}$ and the longevity prone Lister-Albany strain at the NIH by Hansen $^{8}$ thereby establishing the essential criterion for the designation of congenic status. ${ }^{8} 9$ This innovative breeding combination resulted in a unique strain of rat with obese as the only major atypical characteristic, and a longer lifespan than most or all other genetically obese strains, ${ }^{1}$ and thus provide an opportunity to design studies where the only common variables were the lean vs obese phenotype. ${ }^{1,14,24}$

Lean rats of this strain remain physiologically normal throughput their long lifespan, which can exceed 4 years, ${ }^{1}$ while the demonstrated longevity in the obese phenotype may exceed 3 years, almost twice as long as some other strains, including the Zucker fatty rat (fa/ $\mathrm{fa}$ ), the atherogenesis-prone JCR-corpulent rat and others. ${ }^{32-38} \mathrm{In}$ the present study we sought to examine the physiologic course of the and biochemical parameters early during the development of obesity to determine the characteristics of early markers known to impact on established components of energy balance and energy expenditure. Diet induced thermogenesis (DIT) and non-shivering thermogenesis (NST) contribute to the phenomena of adaptive thermogenesis in rodents, where they have been proposed to provide 
an energy buffer for overall energy balance and also contribute to body temperature regulation and homeostasis. ${ }^{5-7,19}$ The current study was conducted in normally reared littermate pairs during the postweaning, preadolescent growth period extending from 6 weeks to 9 weeks of age. Obese animals contain both $-c p$ alleles, while their lean littermates are presumed to have carried one or neither -cp allele, as lean rats containing a single $-c p$ trait (heterozygous lean) or none at all (homozygous lean) are virtually identical in physical appearance and physiologic behavior throughout much of their lifespan in our experience. $^{1,14,24}$

BAT-mediated thermogenesis is normally robust in genetically lean rodents, and typically demonstrates an enhanced response to dietary intervention, noradrenaline (NE) infusion, and to environmental challenge. In contrast, numerous studies have reported that the thermic responses to both diet and environment and $\mathrm{NE}$ are at least partially or significantly impaired in the obese phenotype in this and other forms of obesity in rodents. ${ }^{32-35}$ The age of onset of the thermic impairments is unclear however and is likely modifiable by other developing comorbidities such as NIDDM, hypertension, or development of renal disorders. ${ }^{11,12}$ The epigenetic markers are likely established at or soon after zygotic formation but are not expressed immediately during the preweaning stage of growth. Most studies have been reported in male offspring of various ages, while in the present study young female animals were selected for the study, since less is known about gender-linked differences which may exist. ${ }^{39-43}$ There are clear gender linked differences in lipid accretion between male and female rodents reported, due in part to gender linked differences in fat depots and likely differences in the distribution kinetics, where male offspring appear to develop obesity patterns earlier in life although comparative studies are sparse. In the present study, IBAT mass and thermogenic activity as expressed by T4-5'-deiodinase activity and circulating $\mathrm{T} 3$ concentrations, in combination with measures of $\mathrm{VO} 2$ before and following cold exposure differed significantly between the lean and obese phenotype, suggestive that the primary physiologic impairment may have multiple components, likely at a nuclear receptor, neuroendocrine or intracellular locus independent of the T3 generation mechanisms. In other studies, experimental overnutrition induced-DIT and the thyroidal response to overnutrition were impaired in the obese phenotype in male offspring. Thus, the physiologic mechanisms in DIT vs NST although similar, may make differential contributions to energy homeostasis in this strain of rat, and likely includes contributions from BAT, Beige Brown adipose tissue and other tissues..$^{15,44-48}$ The thermic responses to cold exposure however showed clear differences between lean and obese littermates, with the initial and post shivering phase significantly more robust in the lean in all observations. In ob/ob mice, the obese mice fared poorly when exposed to a cold environment, with many succumbing to the cold. In the current studies, survival of both lean and obese littermates exposed to a $4^{\circ} \mathrm{C}$ cold exposure for up to 14 hours was without any animals succumbing to the cold, and with only modest decreases in peripheral body temperature in the obese rats. The basis for the better survival in the obese rats vs. the obese mice may also reflect the differences in proportional surface area to lean mass, as the lean mass is where much of the heat may be generated and is much smaller in mice than in rats. Longer durations of cold exposure have not been determined in our studies. Fatty tissue while an excellent electrical insulator, has been reported to provide less thermal protection likely due in part to less vascularization and a metabolically less active cytoplasmic mass, much of which may be displaced with lipid deposition and storge in the fatty vs lean tissues. At the rat ages studied in the present study, central fat depots greatly exceeded subcutaneous depots, thus the insulative impact of subcutaneous adipose tissue was likely less important in maintaining temperature homeostasis that may occur in older and fatter obese animals.

In rats and other mammalian species, the majority of the conversion of $\mathrm{T} 4$ to $\mathrm{T} 3$ occurs in peripheral tissues, via one of two T4-5' deiodinase enzymes, Type I and Type II. ${ }^{28-30}$ In the present study, total T4-5'-deiodination of both Type I and Type II deiodinases was measured in the procedure applied. The Type II deiodinase is more prominent in BAT, where it may generate abundant quantities of free T3 for both local and peripheral T3-mediated effects on metabolism and endogenous heat generation when activated by diet or environment, while the Type I deiodinase has been attributed to liver and other tissues and may provide the slower but more consistent contributions to circulating T3 concentrations and endocrinologic feedback regulation. Baseline deiodinase activity in the obese phenotype was more than twice that observed in the lean phenotype at both environmental temperatures studied and may reflect a compensatory response consistent with an altered feedback regulation mechanism in that instance. The circulating T4 concentrations were unaffected by temperature of exposure or phenotype, and the concentration differences between $\mathrm{T} 3$ and T4 likely ensured an abundant substrate supply of the T4 prohormone. Deiodinase activity of liver tissue indicated a significant phenotype effect, with the activity in lean rats consistently greater than in their obese littermates and a temperaturephenotype interaction of unknown origin. The total muscle mass of an animal represents one of the largest overall tissue beds and has been reported to generate between $20 \%$ or more of the circulating T3 in a 'slow' but constant T3 pool. Deiodinase activity in gastrocnemius muscle was lower than observed in other tissues and was unaffected by temperature or phenotype. The generation of T3 from T4 in renal tissue homogenates was also similar in both phenotypes, and only modestly impacted by phenotype but not by cold exposure, indicating that cold was not a factor in mediating net renal T4 5 ' deiodinase activity.

Deiodinase activity also occurs in liver, ${ }^{28}$ where T3 plays a decisive role in the $\alpha$-glycerophosphate shuttle, which is linked to mitochondrial heat generation and a decrease in the efficiency of energy metabolism. Galton and Bray ${ }^{45}$ demonstrated decreased in the $\alpha$-glycerophosphate shuttle in humans, and Tatleman and Tulp ${ }^{46}$ reported that the activity of the shuttle increased in parallel with circulating T3 concentrations in lean, cafeteria-overfed rats. Mitochondria have active binding sites for T3, which results in greater heat generation in proportion to the elevations in T3 availability. Thus, renal tissue provides an ongoing source of peripheral T3 generation to compliment the circulating hormone levels and thus contribute to endocrinologic feedback mechanisms for T3 generation. ${ }^{45-48}$

The other elephant in the room here with respect to thermogenesis is insulin sensitivity vs insulin resistance. Insulin actions contribute significantly to the overall resting metabolic rates via several mechanisms, among the most important being insulin mediated glucose uptake in muscle and other peripheral tissues, and insulin contributions to the economy of protein turnover., ${ }^{1,1720,42}$ Protein turnover is the net of protein synthesis minus protein degradation, at an ATP cost of 4 ATPs per peptide bond formed during the synthesis and elongation of peptides during the formation of tissue proteins. Since protein synthesis represents one of the most energetically expensive biochemical processes in muscle and other tissues, where the rates of protein synthesis are closely matched to the rates of protein degradation, any decrease in the rate of protein degradation or protein synthesis would require less energy input and result in an improved economy of energy metabolism. Protein degradation is a process that is decreased by insulin, while insulin plays a permissive 
role in protein synthesis. Thus, in the presence of hyperinsulinemia or insulin resistance, the net effect of insulin action of muscle would be to decrease the rate of synthesis but not the half-life of tissue proteins. The cumulative effect on energy expenditure would be to become more energetically efficient as less ATP would be needed to support new peptide bond formation. Previous studies in the LA/Ntul//-cp rats support this hypothesis. Insulin has also been shown to be a critical factor in BAT-mediated thermogenesis, where insulin resistance inhibited the thermogenic process in vitro. ${ }^{32-35}$

Finally, the role of insulin actions in lipid metabolism are well established. Insulin not only stimulates de novo lipid synthesis, but also impedes lipid mobilization in white adipose tissues, but not in all adipose tissues equally. Central body fat is more sensitive to insulin-stimulated lipid accretion, where it is associated with greater adverse metabolic sequelae than at least some subcutaneous fat depots. Lower body fat accumulation, particularly in females poses fewer cardiovascular related health complications than upper body fatness. Corresponding comparisons have not been reported in the rat however, as parameters of lipid metabolism including greater HDLcholesterol concentration in most rodent strains than occurs in most humans. The LA/N-cp, SHR/N-cp and the JCR-cp strains appear to be the exceptions among rat strains, as all strains that carry the -cp trait demonstrate variable degrees of hyperinsulinemia and insulin resistance, conducive to formation of atheroma's and more advanced cardiovascular lesions, and which may also contribute to the NE resistance often reported in the $-c p$ strains.

\section{Conclusions}

In conclusion, the current study reports parameters of impaired thermoregulatory responses to otherwise healthy, postweaning preadolescent female obese rats, while the thermoregulatory responses occurred normally in the lean phenotype. In as much as the only known difference between the lean and obese phenotypes of this strain of the expression of the -cp trait, likely via a chronologic inspired epigenetic mechanism with an age of activation of onset sometime during the preweaning growth stage. Detailed DNA studies may identify and resolve those offspring that may be predisposed to develop obesity at a later stage of growth. Because the transition from lean beginnings to preobese offspring occurs gradually and without overt signs, metabolic factors at the neuroendocrine or metabolic level are likely operative in the epigenetic expression of obesity in the corpulent rat.

\section{Acknowledgments}

The author acknowledges Ms. Mary Victor and Huang Peisong for animal care and husbandry, Dr Steve Dubin for veterinary care, and Ms. Deborah A Outtrim (Yarnell) for data collection, analysis, and data processing. The author acknowledges Professors Aftab Awan and George Einstein of USAT and UHHVI for editorial assistance in reviewing this manuscript and data analysis. In addition, the author wants to thank Drexel University, the University of Science Arts and Technology, Montserrat and the University of Health and Humanities, Virgin Islands for Institutional resources to complete these studies.

\section{Conflicts of interest}

The authors declare that they have no competing interests.

\section{Funding}

None.

\section{References}

1. Tulp OL. Characteristics of thermogenesis, obesity, and longevity in the LA/N-cp rat. ILAR News. 1990;32(3):32-39.

2. Michaelis OE IV. New Models of Genetically Obese Rats for Studies in Diabetes, Heart Disease, and Complications of Obesity, Veterinary Resources Branch, Division of Research Services, NIH publication, Bethesda, MD. 1988. p. 13-15.

3. Tulp OL, Einstein GP. Thermogenesis, aging and obesity in the LA/tul//cp (corpulent) rat. Adv Obes Weight Manag Control. 2021:11(2):37-43.

4. Tulp OL, DeBolt SP. Aging and Obesity in the Corpulent Rat. In:Nestle Nutrition Series, Research and Practice in MNA and Aging. 1999. p. 149-155.

5. Himms-Hagen J. Thermogenesis in BAT as an energy buffer. NEJM. 1983;311:1150-1156.

6. Rothwell N J, Stock MJ. A role for brown adipose tissue in diet-induced thermogenesis. Nutrition \& Metabolism. 1997;5(6):650-656.

7. Trayhurn P. Origins and early development of the concept that brown adipose tissue thermogenesis is linked to energy balance and obesity. Biochimie. 2017;134:62-70.

8. Hansen, CT. The development of the SHR/N- and LA/N-cp (Corpulent) Congenic Rat Strains. In:New Models of Genetically Obese Rats for Studies in Diabetes, Heart Disease, and Complications of Obesity. NIH publication, Division of Research Services, Veterinary Resources Branch, National Institutes of Health, Bethesda, MD> 1988. p. 7-10.

9. Koletsky S. Pathologic findings and laboratory data in a new strain of obese hypertensive rats. Am J Pathol. 1975;80(1):129-142.

10. Greenhouse, DD. New Models of Genetically Obese Rats for Studies in Diabetes, Heart Disease, and Complications of Obesity. Ilar News. 1990;21(3) 1-5.

11. EB Kahle, JM Dadgari, GA Dudley, et al. Adaptive Response of Enzymes of Carbohydrate and Lipid Metabolism to Exercise, in New Models of Genetically Obese Rats for Studies in Diabetes, Heart Disease, and Complications of Obesity. Veterinary Resources Branch, Division of Research Services, NIH publication, Bethesda MD. 1988;143-148.

12. Huang HJ, Young AA, Koda, JE, Tulp, OL, Johnson MJ and Cooper GJ. Hyperamylinemia, hyperinsulinemia, and insulin resistance in genetically obese LA/N-cp rats. Hypertension. 1992;19.

13. Michaelis OEIV, Ellwood KC, Tulp OL, et al. Effect of feeding sucrose or starch diets on parameters of glucose tolerance in the $\mathrm{LA} / \mathrm{N}$-corpulent rat. Nutr Res. 1986;6(2):95-99.

14. Tulp OL. Effects of aging, phenotype, and carbohydrate feeding on caloric efficiency and adiposity in the LA/Ntul//- $c p$ (Corpulent) rat. $A d v$ Obes Weight Manag \& Control. 2021:11(1) 5-11.

15. Rahman Michael S, Einstein George P, Tulp Orien L. Autonomic, Immunological and Endocrine influences on adipose tissue as an organ. Adv Obes Weight Manag Control. 2021:11(2):48-58.

16. Tulp OL. XIV Int Congress of Nutrition. 1985.

17. Tulp OL. Sympathetic and Thyroidal Components of Diet-Induced Facultative Thermogenesis in the LA-Corpulent Rat" Brain Res. Bull. $1985 ; 348$.

18. Young NL, Tulp OL. The effects of norepinephrine and nutritional status on resting metabolic rates in $\mathrm{LA} / \mathrm{N}-\mathrm{cp}$ rats. Comp Biochem Physiol. 1989;597-603.

19. Rothwell NJ, Stock MJ. A role for brown adipose tissue in diet-induced thermogenesis. Nutrition \& Metabolism. 1997;15(6):650-656.

20. Tulp OL. Impaired activation of thermogenesis in the corpulent rat. Life Sciences. 1984;35:1699-1705. 
21. Jacks DG, Kerna NA. A Comprehensive Analysis of Obesity Part 1. Overview of Obesity. J Obese Nutr Disord. 2018;JOND-130.

22. Center for Disease Control and Prevention. National Health and Nutrition Survey. (NHANES Data Base, NIH). 2013-2014.

23. Tulp OL, Awan AA, Lewis N, et al. Can epigenetic expression contribute to the development of an obese phenotype? AOWMC. 2021:11:98-101.

24. Tulp OL, Einstein, GP. Thermogenesis, aging and obesity in the LA/tul//cp (corpulent) rat. Adv Obes Weight Manag Control. 2021:11(2):37-43.

25. Wang ZM, Zhang J, Ying Z, et al. Organ-Tissue Level Model of Resting Energy Expenditure Across Mammals:New Insights into Kleiber's Law. Int Scholarly Research Network. IBSN Zoology. 2012:Art ID 673050.

26. Tulp, OL, McKee STD. Triodothyronine (T3) Neogenesis in lean and obese LA/N//-cp Rats. Biochem Biophys Res Commun. 1986;140134142 .

27. Lowry OH, Roseborough NJ, Farr AL, et al. Protein measurement with the Folin-phenol reagent. J Biol Chem. 1951;193:265-275.

28. Young RA, Shih-Lieh F, Prosky J, et al. Hepatic Conversion of thyroxine in obese and lean Zucker rats. Am J Physiol. 1987;252:E63-E67.

29. Tulp OL, McKee STD, Michaelis OE IV. Effects of dietary carbohydrate type on T4-5'-deiodinase activity of rats. FASEB J. 1988;2.

30. Tulp OL Expression of peripheral thyroxine 5' deiodinase activity:a link between the metabolic efficiency of undernutrition and malnutrition and the predisposition toward the development of obesity. Prog. 11 Interamerican conference on health education. Mexico City. 1984.

31. Ott L. Multiple comparisons. In:An Introduction to Statistical Methods and Data Analysis. $3^{\text {rd }}$ ed. PWS-Kent, Boston MA. 1988;437-466.

32. Marette A, Tulp OL and Bukowiecki, LJ. Mechanism linking insulin resistance to defective thermogenesis in brown adipose tissue of obese diabetic SHR/N -cp rats. Int J Obese. 1991;15:23-831.

33. Marette A, Maurige P, Bukowiecki LJ, et al. Hormonal regulation of brown adipocyte glucose transport in genetic obesity. In:Obesity:Dietary factors and Control, D.R. Romsos, editor. Basel: Karger, 1991;119-130.

34. Atgie C, Marette A, Desaultels M, et al. Specific decrease in mitochondrial thermogenic capacity in brown adipose tissue of obese SHR/N-cp rats. Am J Physiol. 1993;34:1674-1680.

35. Abdoulaye D, Pierce WD, Kelly SE. et al. Abnormal insulin and glucose metabolism in the JCR:LA-corpulent rat. Front Nutr. 2016;3:44.
36. Tulp, OL Stevens, C Barbie OA Non-shivering thermogenesis and obesity in the adult diabetic Wistar fatty rats. Comp Physiol Pharm.1989;92A(1):37-41.

37. Tulp, OL OE Michaelis IV, Servetnick, DA, Hansen, CT, and Black, DE. Nonshivering thermogenesis and obesity in the diabetic SKY/N $-\mathrm{cp}$ rat. Comp Biochem Physiol. 1994;107A:195-201.

38. Young, RA, Tulp, OL, Horton ES. Thyroid and growth responses of young Zucker obese and lean rats to a low protein-high carbohydrate diet. J Nutr. 1980;110:1421-1431.

39. Danforth E Jr. The role of thyroid hormones and insulin in the regulation of energy metabolism. Am J Clin Nutr. 1983;38:1006-1017.

40. Danforth E. Diet and Obesity. Am J Clin Nutr. 1985;41:1131-1145.

41. Trayhurn P. Origins and early development of the concept that brown adipose tissue thermogenesis is linked to energy balance and obesity. Biochimie. 2017;134:62-70.

42. Tulp OL, Black DE, Outtrim D, et al. Peripheral thyroid hormone metabolism and actions (THMA) in lean and obese LA/Ntul//-cp rats. FASEB J. 1994.

43. Haffner SM. Sex hormones, obesity, fat distribution, type diabetes, and insulin resistance:epidemiological and clinical correlation. Int $J$ Obes. 2000;24:556-558.

44. McCann UD, Shaw EA, Kaplan MM. Iodothyronine deiodination reactions in several rat tissues. Effects of age, thyroid status, and glucocorticoid treatment. Endocrinology. 1984;114:1513-1521.

45. Galton DA, Bray G. Metabolism of $\alpha$-Glycerol Phosphate in Human Adipose Tissue in Obesity. The Journal of Clinical Endocrinology \& Metabolism. 1967;27(11):1573-1580.

46. Tulp OL. Effect of $\alpha$-methylparatyrosine on thermogenesis in lean and corpulent rats. Clin Res. 1984;32:237A.

47. Tatleman HM, Tyzbir RS, Tulp OL. Effects of overfeeding on BAT and liver mitochondrial metabolism and shuttle activity in adult rats. Fed Proc. 1981;40:871.

48. Labros Sidossis L, Shingo Kajimura S. Brown and beige fat in humans:thermogenic adipocytes that control energy and glucose homeostasis. J Clin Invest. 2015;125(2):478-486. 